Mai 2002
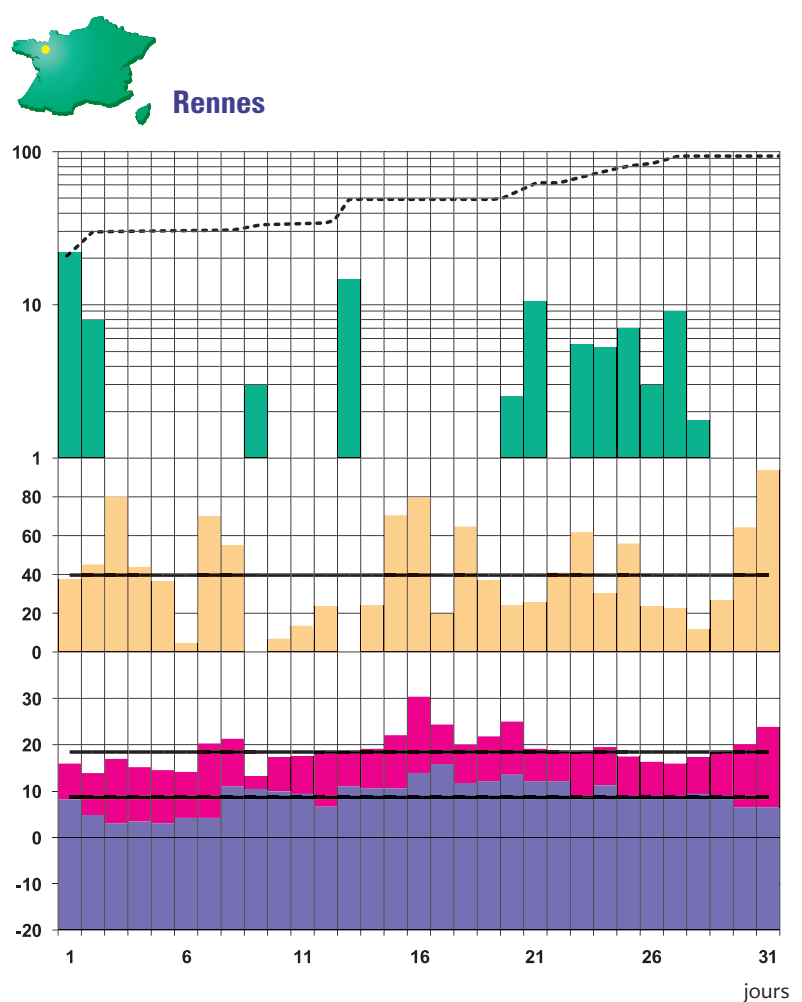

Total mensuel des précipitations : 1,5 fois la normale

Fraction d'insolation moyenne : normale

Température moyenne : $>0,3^{\circ} \mathrm{C}$ à la normale
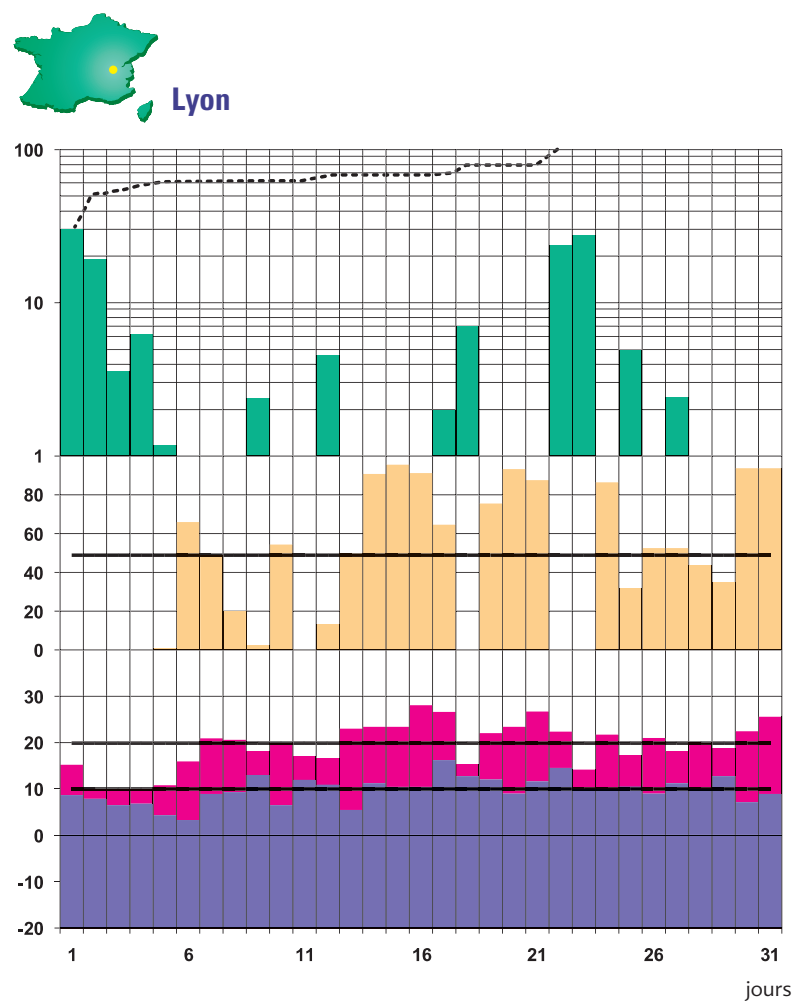

Total mensuel des précipitations : 1,6 fois la normale Fraction d'insolation moyenne : légèrement déficitaire Température moyenne : $<0,4{ }^{\circ} \mathrm{C}$ à la normale

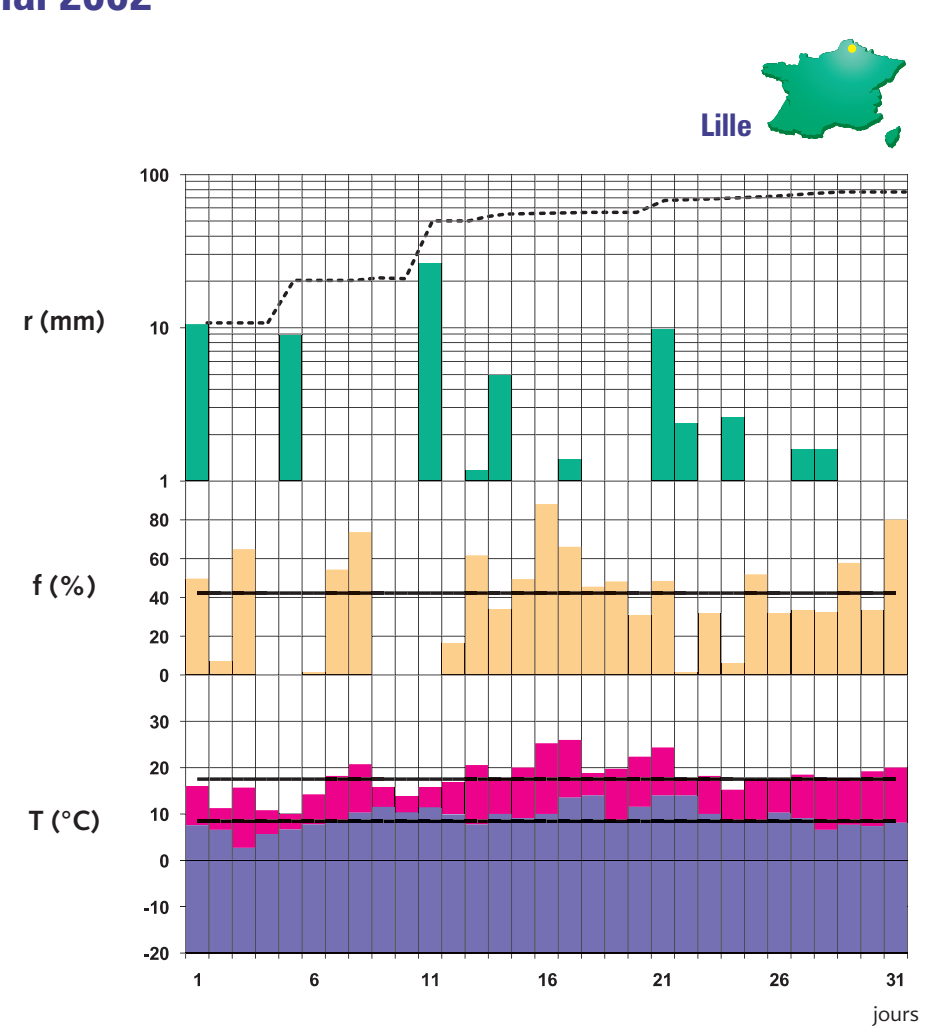

Total mensuel des précipitations : 1,2 fois la normale

Fraction d'insolation moyenne : légèrement déficitaire

Température moyenne : $>0,5^{\circ} \mathrm{C}$ à la normale
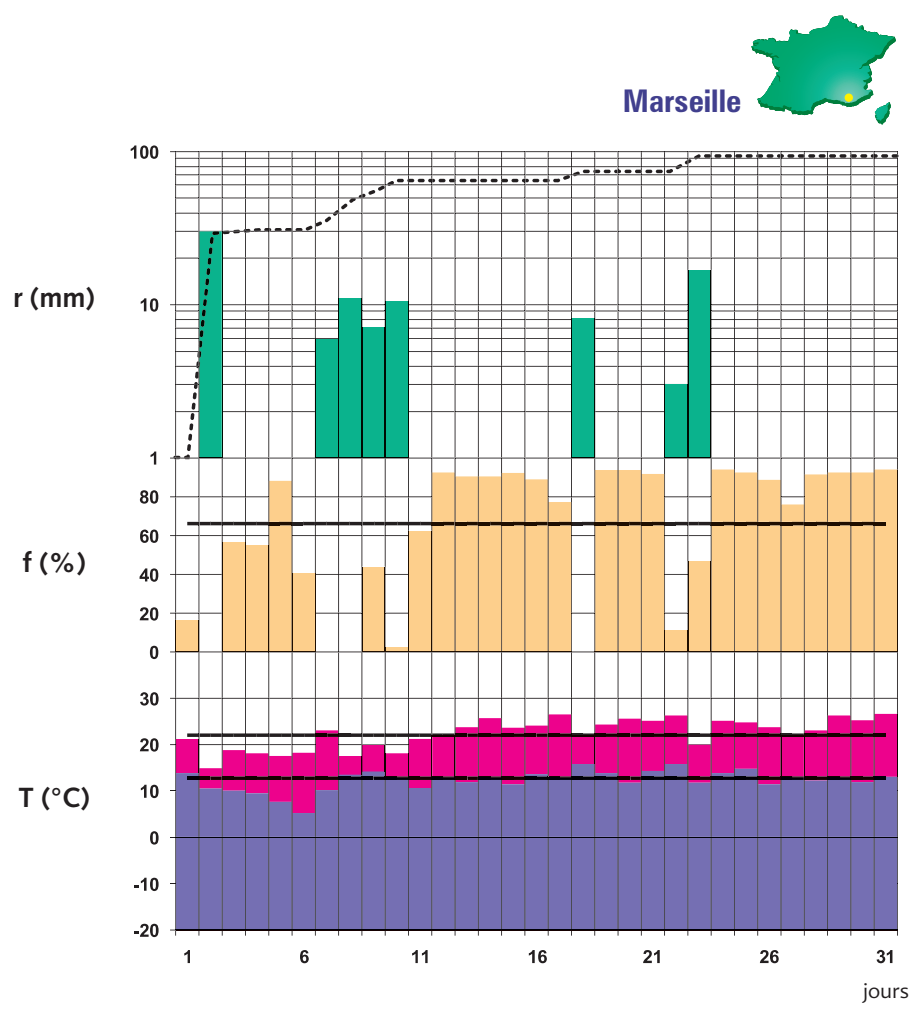

Total mensuel des précipitations : 2,3 fois la normale

Fraction d'insolation moyenne : normale

Température moyenne : $<0,1^{\circ} \mathrm{C}$ à la normale

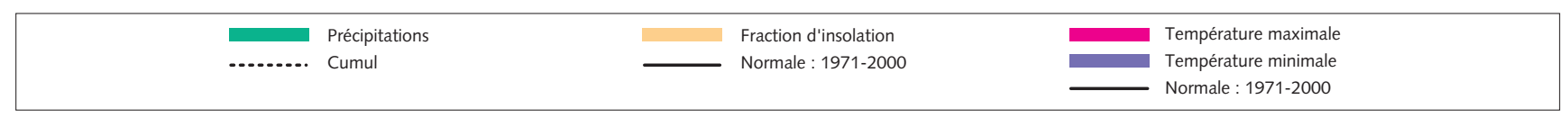




\section{Mai 2002}
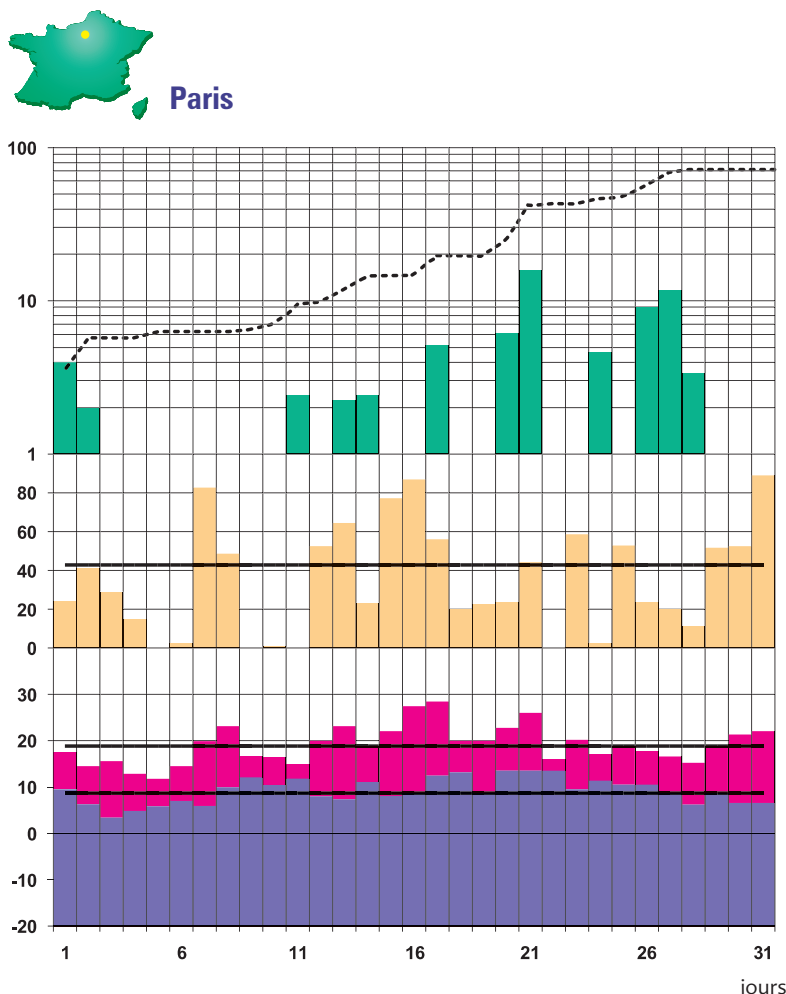

Total mensuel des précipitations : 1,2 fois la normale Fraction d'insolation moyenne : légèrement déficitaire Température moyenne : $>0,3^{\circ} \mathrm{C}$ à la normale
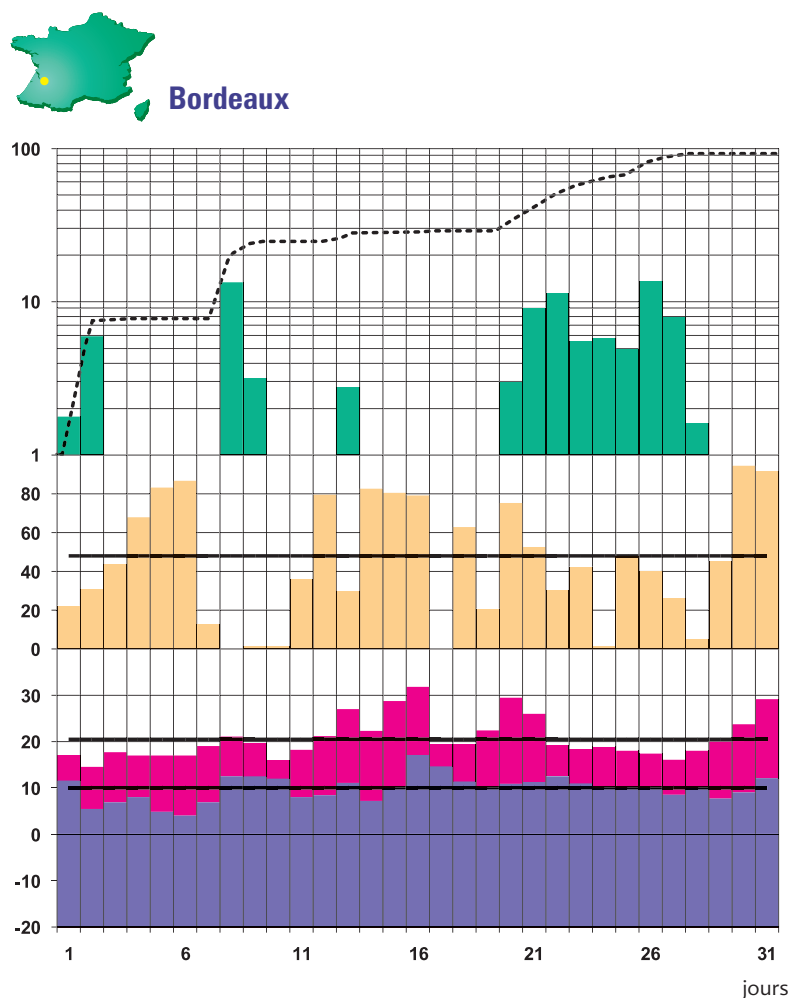

Total mensuel des précipitations : 1,1 fois la normale

Fraction d'insolation moyenne : normale

Température moyenne : $<0,1^{\circ} \mathrm{C}$ à la normale

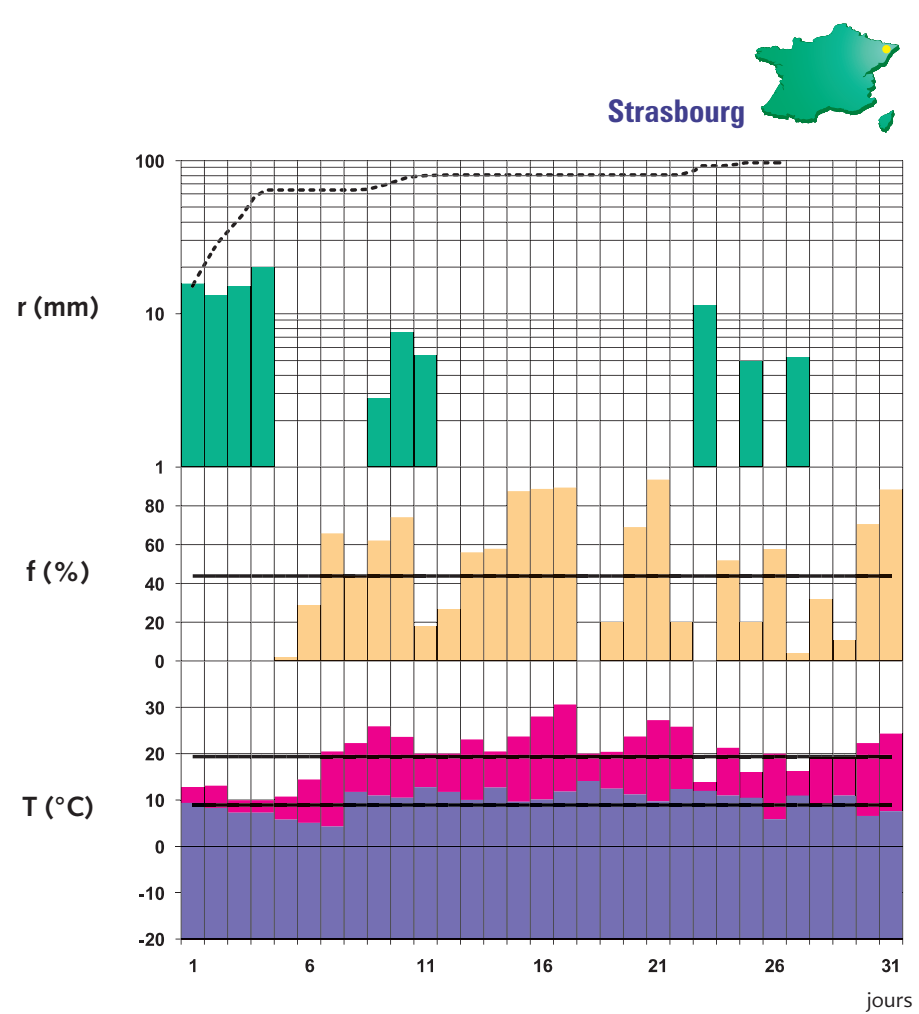

Total mensuel des précipitations : 1,3 fois la normale

Fraction d'insolation moyenne : normale

Température moyenne : $>0,6^{\circ} \mathrm{C}$ à la normale

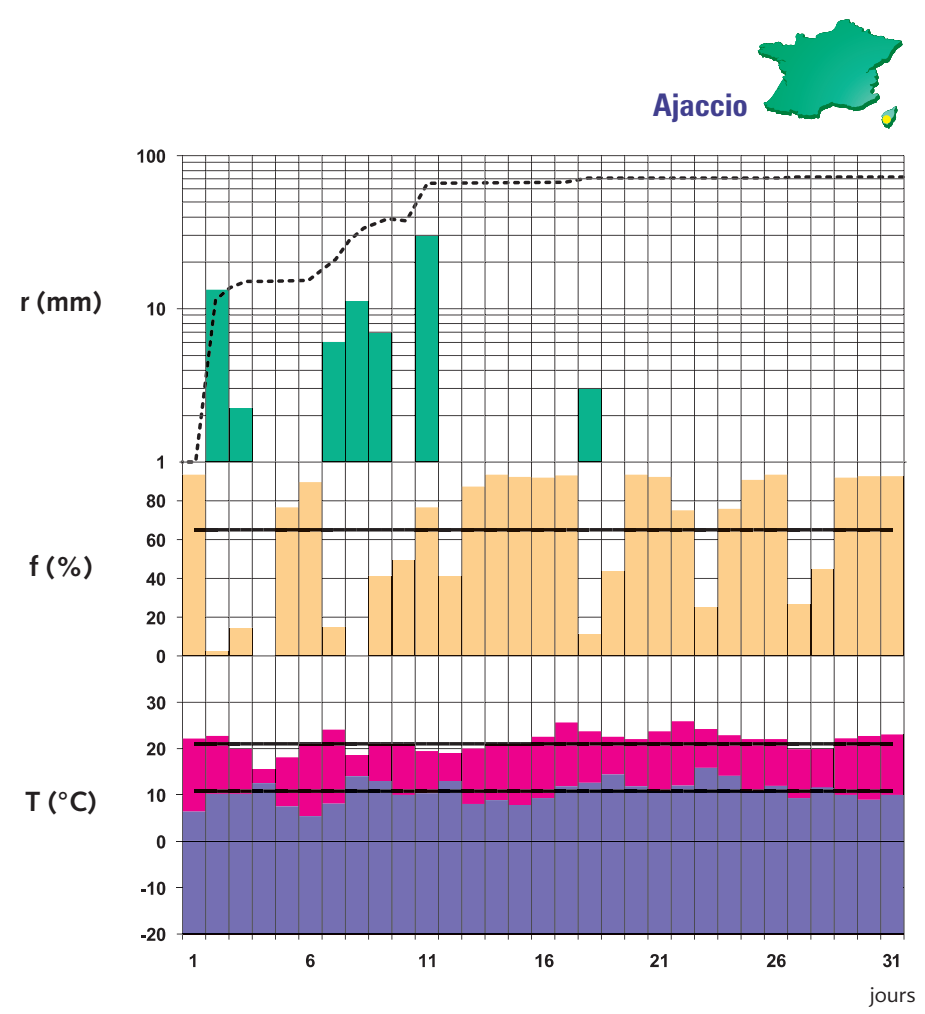

Total mensuel des précipitations : 1,8 fois la normale

Fraction d'insolation moyenne : normale

Température moyenne : $>0,3{ }^{\circ} \mathrm{C}$ à la normale

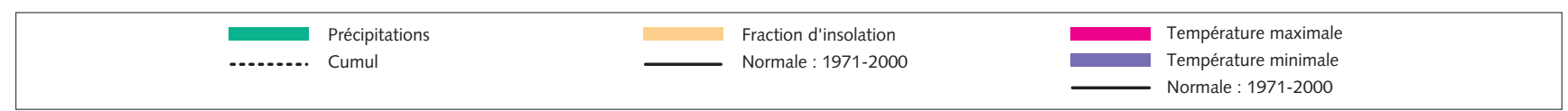




\section{Mai 2002}

Heures UTC sur tout le bulletin

\section{Périodes thermiques}

\section{De la fraîcheur en première décade,} de fortes chaleurs en deuxième.

\section{Première décade}

Minimales. Si les valeurs sont de saison le $1^{\text {er }}$, avec la totalité des postes qui enregistre au moins $5{ }^{\circ} \mathrm{C}$, il n'en est pas de même dès le 2 où une advection d'air frais dans les basses couches fait descendre le thermomètre, tout d'abord sur l'Ouest : $14,6{ }^{\circ} \mathrm{C}$ à Montpellier (Hérault) le $1^{\text {er }}$; $0,8^{\circ} \mathrm{C}$ à Lapouyade (Gironde) le 2.

La fraîcheur s'accentue jusqu'au 6 et l'on observe dès le 3 de petites gelées; le 6, elles sont nombreuses des Landes au plateau de Langres. Durant cette période, un petit quart sud-est, la Corse, la vallée du Rhône ainsi qu'une zone qui s'étend des Flandres à l'Alsace restent souvent à l'écart de cette chute du mercure : $-0,4{ }^{\circ} \mathrm{C}$ à Romorantin (Loir-et-Cher) et $15,5^{\circ} \mathrm{C}$ à Ajaccio (Corse-du-Sud) le 3 ; $-2,8{ }^{\circ} \mathrm{C}$ à Aubusson (Creuse) le 6.

À partir du 7, la tendance s'inverse et les fins de nuit deviennent plus clémentes ; du 8 au 10, les minimales passent audessus des normales sur la quasi-totalité du pays et le 9 , journée la plus chaude de la décade, $90 \%$ des postes dépassent les $10{ }^{\circ} \mathrm{C}: 13,7{ }^{\circ} \mathrm{C}$ à Saint-Yan (Saône-etLoire) [normale $7,7^{\circ} \mathrm{C}$ ] le $9 ; 13,1^{\circ} \mathrm{C}$ à Charleville-Mézières (Ardennes) [normale $6,9^{\circ} \mathrm{C}$ ] le 10.

Maximales. Le mois débute dans la fraîcheur, avec des maximales sous les normales saisonnières sur la majeure partie du territoire jusqu'au 5 ; la façade est enregistre les valeurs les plus basses : 9,8 ${ }^{\circ} \mathrm{C}$ à Mâcon (Haute-Saône) [normale 19,5 ${ }^{\circ} \mathrm{C}$ le le $1^{\text {er }}$; 7,7 ${ }^{\circ} \mathrm{C}$ à Chambéry-Aix (Savoie) [normale $20,2{ }^{\circ} \mathrm{C}$ ] le $4 ; 7,8^{\circ} \mathrm{C}$ à Saint-Dizier (Haute-Marne) [normale $\left.19,3{ }^{\circ} \mathrm{C}\right]$ le 5 .

À partir du 6, la douceur envahit progressivement le pays, les valeurs inférieures à $10{ }^{\circ} \mathrm{C}$ sont rares. Des poches d'air à plus de $20{ }^{\circ} \mathrm{C}$ se forment le 7 du Sud-Est à la Beauce, le 8 de l'estuaire de la Gironde à l'Anjou et aux frontières du nord-est, le 9 sur un petit quart nord-est ainsi que sur le Quercy et la Corse, le $10 \mathrm{du}$ Bourbonnais à la Lorraine et à l'Alsace ; durant ces quelques jours, les Pyrénées restent sous les normales saisonnières : $12,3{ }^{\circ} \mathrm{C}$ à
Tarbes-Ossun (Hautes-Pyrénées) le 7 ; 12,8 ${ }^{\circ} \mathrm{C}$ à Saint-Paul-de-Fenouillet (Pyrénées-Orientales) et $27{ }^{\circ} \mathrm{C}$ à Thouars (Deux-Sèvres) le $8 ; 28,4{ }^{\circ} \mathrm{C}$ à Neuvizy (Ardennes) le 9.

\section{Deuxième décade}

Minimales. Les fins de nuit sont encore agréables le 11 et dépassent les normales sur le nord du pays. Le 12, la douceur se déplace sur l'est, alors que quelques valeurs, fraîches pour la saison, sont présentes sur la moitié ouest : $12,6{ }^{\circ} \mathrm{C}$ à Avize (Marne) le $11 ; 2,9{ }^{\circ} \mathrm{C}$ à Trémeur (Côtes-d'Armor) et $12,8^{\circ} \mathrm{C}$ à Lauterbourg (Bas-Rhin) le 12.

Le temps fortement perturbé du 13 sur une petite moitié ouest permet au mercure de remonter, tandis que des monts de Lacaune à la Brie et au Diois, il chute de manière très sensible, de faibles gelées étant même enregistrées : $-0,1^{\circ} \mathrm{C}$ à Aubusson (Creuse) et $12,7{ }^{\circ} \mathrm{C}$ à la Pointe-de-Socoa (Pyrénées-Atlantiques). Même si l'on ne mesure plus de valeurs négatives, la fraîcheur persiste le 14 de la Bigorre à la Côte d'Or, le 15 de la Vendée aux monts du Forez et au plateau de Langres ; en revanche, de la Bretagne au Nord, en Alsace et dans la vallée du Rhône, on est au-dessus des normales. Le 16 sur la moitié ouest et les 17 et 18 sur la quasi-totalité du pays, le thermomètre grimpe fortement: $16,2{ }^{\circ} \mathrm{C}$ à Saint-Girons (Ariège) [record depuis 1949] et $17,7^{\circ} \mathrm{C}$ à Limoges (HauteVienne) [ancien record $17,5^{\circ} \mathrm{C}$ ] le 16 ; $19{ }^{\circ} \mathrm{C}$ à Boulogne (Pas-de-Calais) [normale $9^{\circ} \mathrm{C}$ le 17

La grande douceur de ces trois derniers jours perd du terrain les 19 et 20 et une bonne moitié des stations ne franchit plus les $10{ }^{\circ} \mathrm{C}$.

Maximales. Si les deux premiers jours de la décade sont plutôt frais pour la saison, mis à part pour quelques régions du Sud, le mercure monte en flèche le 13 sur la quasi-totalité du territoire et l'on franchit souvent les $25{ }^{\circ} \mathrm{C}$ dans le Sud-Ouest : $10,5^{\circ} \mathrm{C}$ à Ahun (Creuse) le $11 ; 29,2{ }^{\circ} \mathrm{C}$ à Sabres (Landes) et $30,8^{\circ} \mathrm{C}$ à Pau (Pyrénées-Atlantiques) le 13

Un temps de traîne le 14 fait redescendre dans le Nord les maximales à un niveau de saison ; mais, dès le 15 , elles remontent sur tout le pays pour atteindre parfois des records les 16 et $17: 28,1{ }^{\circ} \mathrm{C}$ à Saint-Sylvain (Calvados) [ancien record $26,3{ }^{\circ} \mathrm{C}$ ], $34,2{ }^{\circ} \mathrm{C}$ à Biscarrosse (Landes) [ancien record $33,1^{\circ} \mathrm{C}$ ] et $35,7^{\circ} \mathrm{C}$ à Carcans (Gironde) le $16 ; 28,7{ }^{\circ} \mathrm{C}$ à Roville-aux-Chênes (Vosges) [ancien record 28,6 ${ }^{\circ} \mathrm{Cl}$ et $30,4{ }^{\circ} \mathrm{C}$ à Chambon-sur-Voueize (Creuse) [ancien record $29,9^{\circ} \mathrm{C}$ ] le 17.
La fraîcheur arrivée le 17 sur la moitié ouest gagne rapidement tout le pays le 18 pour reculer à nouveau les 19 et 20 : $12,8{ }^{\circ} \mathrm{C}$ à Cellieu (Loire) le $18 ; 32,3{ }^{\circ} \mathrm{C}$ au Temple (Gironde) le 20.

\section{Troisième décade}

Minimales. Les fins de nuit sont à nouveau plus chaudes le 21 , mais aussi et surtout le 22 où près de $100 \%$ des postes enregistrent plus de $10{ }^{\circ} \mathrm{C}$ : $18{ }^{\circ} \mathrm{C}$ au Cap-Corse (Haute-Corse) le 22 .

Le mercure descend ensuite pour se maintenir autour des normales du 23 au 29 avec des minimales le plus souvent comprises entre 10 et $15^{\circ} \mathrm{C}$ sur le Midi, entre 5 et $10{ }^{\circ} \mathrm{C}$ sur le reste du territoire. Toutefois, des poches d'air supérieur à $10{ }^{\circ} \mathrm{C}$ sont présentes sur le Nord-Est les 23, 25 et 29, alors que du 26 au 28 , on trouve des valeurs qui sont inférieures à $10{ }^{\circ} \mathrm{C}$ sur quelques régions de 1'Est : $19,1{ }^{\circ} \mathrm{C}$ au CapCorse (Haute-Corse) le $23 ; 1,6{ }^{\circ} \mathrm{C}$ à Die (Drôme) le $26 ; 2,2{ }^{\circ} \mathrm{C}$ à Maxey (Meuse) le 27 ; 17,2 ${ }^{\circ} \mathrm{C}$ à Saint-Tropez (Var) le 29.

La fraîcheur gagne sur une très grande partie nord de la France le 30 et plus d'un quart des stations ne franchit pas les $5{ }^{\circ} \mathrm{C}: 0,8^{\circ} \mathrm{C}$ à Aubusson (Creuse) et $1,3{ }^{\circ} \mathrm{C}$ à Juzennecourt (HauteMarne).

Le 31, le thermomètre gagne quelques degrés, mais de nombreuses valeurs restent encore sous les normales saisonnières.

Maximales. Un petit effet de dorsale permet au soleil de briller le 21 sur la majeure partie du pays et donc aux maximales d'être très élevées, sauf sur la Bretagne : 30, $1^{\circ} \mathrm{C}$ à Montluçon (Allier). Le 22, une brutale chute du thermomètre n'épargne qu'une petite moitié est ; la zone la plus froide s'étend de la Guyenne aux Ardennes : $11,3{ }^{\circ} \mathrm{C}$ à $\mathrm{La}$ Souterraine (Creuse) [ancien record $\left.13,2{ }^{\circ} \mathrm{C}\right]$.

La langue de froid se déplace le 23 vers l'est et court des Alpes du Nord à l'Alsace : $10,5{ }^{\circ} \mathrm{C}$ à Saint-Claude (Jura).

Si le 24 les maximales remontent au sud d'un axe Cazaux-Phalsbourg, elles redescendent dès le lendemain ; seul un petit quart sud-est, auquel vient se joindre un petit quart sud-ouest le 28 , dépasse alors les $20{ }^{\circ} \mathrm{C}$ jusqu'au 29 : $11,1{ }^{\circ} \mathrm{C}$ au Gast (Calvados) le 28 ; $29,2{ }^{\circ} \mathrm{C}$ à Hyères (Var) le 29.

La fin du mois connaît une forte remontée du thermomètre sur la quasitotalité du pays. Il fait chaud le 31 au sud du $45^{\mathrm{e}}$ parallèle : $31,6{ }^{\circ} \mathrm{C}$ à Gignac (Hérault). 


\section{Périodes pluviométriques}

\section{Début de mois copieusement arrosé.}

\section{Première décade}

Une limite intéresse le pays, en particulier l'Est jusqu'au 3, avec des précipitations très soutenues. Elle ondule le $1^{\text {er }}$ des Pyrénées aux frontières de l'Est, laissant des orages locaux éclater à l'arrière. La situation n'évolue guère le 2 : le front reste bloqué sur la façade est ; la traîne reste active et chargée des Pyrénées à la Bretagne et de la Normandie au Nord : $51,5 \mathrm{~mm}$ à Saint-Julien (Jura) et $61 \mathrm{~mm}$ à Montjoux (Drôme) le $\mathrm{I}^{\text {er }}$; $95,9 \mathrm{~mm}$ à Valensole (Alpes-de-Haute-Provence) et 99,2 mm à Saint-Julien (Var) le 2.

Les pluies continuent le 3 du Vaucluse à l'Alsace et les averses parfois orageuses se produisent au sud de l'Aquitaine et de Midi-Pyrénées ainsi que du pays nantais à la baie de Somme : 40 mm à Chatte (Isère). La situation des 4 et 5 est pilotée par la présence d'une goutte froide d'altitude centrée sur notre pays. L'instabilité sévit toujours le 4 sur le quart sud-ouest et des remontées pluvieuses affectent une zone qui s'étend du Lyonnais au nord de la Lorraine. Le temps demeure maussade le 5 avec des pluies plus faibles prenant parfois un caractère d'averses du Nord aux Alpes : 23,8 mm à Poucharramet (HauteGaronne) et $35 \mathrm{~mm}$ à WangenbourgEngenthal (Bas-Rhin) le 4.

Après une accalmie, de petites ondées ont lieu sur le piémont pyrénéen et des pluies instables arrivent dans la nuit du 6 au 7 au nord de la Somme. Puis, le 7, si une nette amélioration a lieu sur le nord du pays, un minimum de surface accompagné de pulsations chaudes provoque des précipitations sur le Sud, temporairement modérées autour du golfe du Lion. Elles s'étendent le 8 vers la Corse, se renforçant avec l'advection d'air chaud : $67 \mathrm{~mm}$ à Eus (Pyrénées-Orientales) le 7 ; 51,4 mm à Solenzara (Corse-du-Sud) et 87,5 mm à Eus (Pyrénées-Orientales) le 8.

L'activité pluvio-instable de la perturbation remonte vers le nord le 9 ; les averses, localement orageuses, sont fréquentes dans le Sud-Est ainsi que le long d'une bande qui s'étire du sud de l'Aquitaine aux Ardennes : $60 \mathrm{~mm}$ à Campistrous (Hautes-Pyrénées), 72,4 mm à la Pointede-Socoa (Pyrénées-Atlantiques), $88 \mathrm{~mm}$ à Cogolin (Var) et 96,8 $\mathrm{mm}$ à SaintVallier-de-Thiey (Alpes-Maritimes).

La goutte froide est toujours présente le 10 sur le Sud-Est. Une limite froide donne des pluies instables en Provence et les précipitations, souvent fortes sous la limite qui stationne sur la France, n'épargnent que le littoral atlantique et le nord de la Seine : 70,2 $\mathrm{mm}$ à Ourouer-lesBourdelins (Cher) et 95,6 mm à SaintVallier-de-Thiey (Alpes-Maritimes).

\section{Deuxième décade}

Des averses se produisent un peu partout le 11, des Pyrénées à la Bretagne et au Nord ainsi que sur toute la façade est. La zone pluvieuse la plus marquée se situe sur un petit quart nord-est avec des orages en Lorraine : 43,6 mm à Phalsbourg (Moselle).

Des orages éclatent en Corse dans la nuit : 29,4 mm à Ajaccio (Corse-du-Sud).

L'instabilité persiste le 12 au nord du $45^{\mathrm{e}}$ parallèle avec des orages localisés : 28 mm à Bourbon-Lancy (Saône-et-Loire). Une limite active et instable avec une nouvelle fois des orages concerne les deux tiers nord-ouest du pays le 13 ; elle donne encore de fréquentes averses de la Bretagne à la frontière belge le 14 : 29,6 mm à Boulogne-surMer (Pas-de-Calais) le 13 ; 23,2 $\mathrm{mm}$ à La Chapelle (Ardennes) le 14.

La hausse de champs se poursuit. La dorsale d'altitude rejette le flux perturbé au nord du territoire le 15. Mais des orages éclatent à nouveau sur la Bretagne le 16. Le flux cyclonique de secteur sud concerne la moitié occidentale du pays le 17. Les pluies modérées qui accompagnent la perturbation sont parfois associées à des orages ; elles sont fréquentes des Pyrénées au Limousin et sur la Bretagne. La perturbation se décale le 18 sur la moitié est : 32,6 mm à Dourgne (Tarn) et 37,4 mm à Brignogan (Finistère) le 17 ; 27,6 mm à Pontarlier (Doubs) et 34,2 mm à Mimet (Bouches-du-Rhône) le 18.

Une nouvelle perturbation arrive par l'ouest le 19 ; mais, dans des champs élevés, elle ne donne que de faibles pluies. En revanche, des averses orageuses se produisent encore sur le Sud-Est : 16,4 mm à Figari (Corse-duSud) avec de l'orage à la station.

La dorsale s'installe sur l'Est le 20. À l'arrière, la masse d'air est instable avec une ligne d'orages qui s'étire du Pays basque à la Basse-Normandie : 18,4 $\mathrm{mm}$ à Caen-Carpiquet (Calvados) avec de l'orage.

\section{Troisième décade}

Une limite arrivée en cours d'aprèsmidi du 21 donne des pluies sur la Bretagne qui se décalent rapidement sur la moitié ouest : $24,5 \mathrm{~mm}$ à $\mathrm{La}$ Péruse (Charente).

Le front progresse le 22 dans un flux de sud à sud-ouest; le corps pluvieux est actif des Pyrénées au nord de la Seine et progresse très lentement vers l'est : 67 mm à Barbentane (Bouches-du-Rhône) et 68,5 mm à Antraigues (Ardèche).
Stationnaire le 23 sur la façade est, il reste très actif : 34,6 mm à Altkirch (Haut-Rhin) et 56,4 mm à Canjuers (Var).

Les pluies sur le Finistère le 23 accompagnent une nouvelle onde qui progresse sur une large moitié ouest le 24 , active dès le matin de la Bretagne au Nord et sur le quart sud-ouest l'après-midi : 20,6 $\mathrm{mm}$ à Villefranchede-Lauragais (Haute-Garonne) le 23.

Quelques résidus affectent encore l'Est le 25 , alors qu'une nouvelle perturbation arrive par le nord-ouest. Tandis que des orages d'air froid éclatent après son passage le 26, des averses persistent jusque dans la nuit sur la moitié ouest : 23,6 mm à Pont-Hébert (Manche) et 29,5 $\mathrm{mm}$ au Barp (Gironde) le 26.

La limite quitte le pays par l'est. La traîne s'active fortement avec de fréquentes ondées le $27: 21,2 \mathrm{~mm}$ avec de l'orage à Paris-Montsouris (Paris) et $37 \mathrm{~mm}$ à Lacaune (Tarn).

Une perturbation pénètre sur une large moitié nord dans un flux zonal le 28, mais les précipitations restent faibles. $\mathrm{La}$ dorsale se développe le 29, les averses du Cotentin aux Flandres et les ondées des Landes au Gers demeurent faibles. Les pressions grimpent ; seules quelques rares ondées débordent des Pyrénées le 31 et des orages éclatent sur le Luchonnais.

\section{La température}

La température moyenne du mois varie de $11,8{ }^{\circ} \mathrm{C}$ à Rostrenen (Côtesd'Armor) à $17,9{ }^{\circ} \mathrm{C}$ au Cap-Corse (Haute-Corse).

L'écart à la normale varie de $-1,8^{\circ} \mathrm{C}$ à Bergerac (Dordogne) à $1,6{ }^{\circ} \mathrm{C}$ à la pointe de la Hague (Manche).

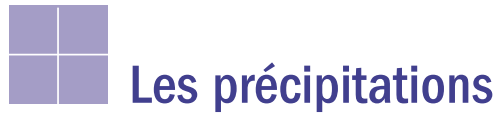

Le cumul mensuel varie de $21 \mathrm{~mm}$ à Cazaux (Gironde) à 288,2 mm à SaintVallier-de-Thiey (Alpes-Maritimes). Le rapport à la normale varie de moins de $30 \%$ à Cazaux (Gironde) à près de $380 \%$ à Saint-Julien (Var).

Le nombre de jours avec précipitations supérieures ou égales à $1 \mathrm{~mm}$ varie de 6 à Cazaux (Gironde) à 20 à Anglet (Pyrénées-Atlantiques).

L'écart à la normale varie d'un déficit de 5 jours à Cazaux à un excédent de 7,4 jours à Mont-de-Marsan (Landes). 


\section{L'ensoleillement}

La durée d'insolation du mois varie de 141,8 heures à Rostrenen (Côtesd'Armor) à 306 heures à Toulon (Var).

\section{Le vent}

\section{Une courte et brutale tempête le 13.}

Le $1^{\text {er }}$, une advection d'air froid renforce nettement sur le nord de la France une traîne au sein de laquelle les fortes rafales sont nombreuses : $86 \mathrm{~km} / \mathrm{h}$ à la Pointe-de-Chémoulin (Loire-Atlantique) et $94 \mathrm{~km} / \mathrm{h}$ à l' 1 le-de-Groix (Morbihan).

Un épisode de mistral et tramontane concerne le pourtour méditerranéen du 3 au 5 ; le 4 , un vent de secteur ouest sur la Corse se joint à eux : 119 et $115 \mathrm{~km} / \mathrm{h}$ au Cap-Béar (Pyrénées-Orientales) les 3 et 4 ; 104 km/h au Cap-Cépet (Var) le 4 ; 122 et $112 \mathrm{~km} / \mathrm{h}$ au Cap-Corse (HauteCorse) les 4 et 5 .

Par ailleurs, un système perturbé génère quelques rafales sur la façade ouest les 3 et 4, sur le nord le $5: 94 \mathrm{~km} / \mathrm{h}$ à la pointe du Raz (Finistère) le 3 ; 94 km/h à PauUzein (Pyrénées-Atlantiques) le 4 ; $90 \mathrm{~km} / \mathrm{h}$ à Dieppe (Seine-Maritime) le 5. Les 7 et 8 , le vent d'est devient très fort du littoral varois à la Corse : $104 \mathrm{~km} / \mathrm{h}$ à L'TlleRousse (Haute-Corse) le 7 ; 108 et
101 km/h à l'île-du-Levant (Var) les 7 et 8. Mis à part quelques rafales côtières ne dépassant pas les $100 \mathrm{~km} / \mathrm{h}$ les 9 et 12 , le vent se fait discret durant ces quatre jours. Il se réveille pour souffler en tempête le 13 du Béarn à la frontière belge, généré par une perturbation active ; dans le Sud-Ouest, les violentes rafales déracinent des arbres dont certains coupent des lignes électriques et arrachent des poteaux téléphoniques. La différence de température entre la côte $\left(14{ }^{\circ} \mathrm{C}\right)$ et la terre (de 26 à $28^{\circ} \mathrm{C}$ ), le vent de secteur sud qui s'oriente à l'ouest au passage du front chaud, sa brusque montée en puissance à ce moment-là pendant 15 à 30 minutes et les précipitations qui restent modérées sont caractéristiques de la galerne. Toutefois, ce terme est abusif car la zone touchée est bien plus importante que pour les galernes classiques (zone côtière du sud-est du golfe de Gascogne) : $104 \mathrm{~km} / \mathrm{h}$ à Jamericourt (Oise) ; 104 km/h à Lille-Lesquin (Nord); $108 \mathrm{~km} / \mathrm{h}$ à Nuaille (Charente-Maritime); $108 \mathrm{~km} / \mathrm{h}$ au sommet de la tour Eiffel (Paris) ;112 km/h à Ouessant-Stiff (Finistère) ; $115 \mathrm{~km} / \mathrm{h}$ à Biarritz (Pyrénées-Atlantiques); $115 \mathrm{~km} / \mathrm{h}$ à Niort (Deux-Sèvres); $119 \mathrm{~km} / \mathrm{h}$ à la Pointe-deSocoa (Pyrénées-Atlantiques) ;126 km/h à Lège-Cap-Ferret (Gironde); $133 \mathrm{~km} / \mathrm{h}$ à Biscarrosse (Pyrénées-Atlantiques)

La traîne de la perturbation précédente n'occasionne plus le lendemain qu'une bourrasque à plus de $80 \mathrm{~km} / \mathrm{h}$ : $86 \mathrm{~km} / \mathrm{h}$ à Dunkerque (Nord) le 14.

Un épisode de marin et d'autan s'installe alors les 16 et 17 ; le 17 , le vent de sud est également soutenu le long de la vallée du Rhône : $101 \mathrm{~km} / \mathrm{h}$ à Labruguière
(Tarn) le $16 ; 86 \mathrm{~km} / \mathrm{h}$ à Lyon-Bron (Rhône), $90 \mathrm{~km} / \mathrm{h}$ à Villefranche-deLauragais (Haute-Garonne), $94 \mathrm{~km} / \mathrm{h}$ au Cap-Béar (Pyrénées-Orientales), $94 \mathrm{~km} / \mathrm{h}$ à Leucate (Aude) et $108 \mathrm{~km} / \mathrm{h}$ à Labruguière (Tarn) le 17.

La tramontane le 18, accompagnée du mistral le 19, intéresse le pourtour méditerranéen : $119 \mathrm{~km} / \mathrm{h}$ au CapBéar (Pyrénées-Orientales) le 18.

Un minimum d'altitude, centré à l'ouest de l'Irlande les 21 et 22 engendre un flux de secteur sud sur le pays ; quelques fortes rafales, parfois accompagnées d'orages, sont mesurées sur les régions proches de la Manche, dans la vallée du Rhône ainsi que des hauteurs du Tarn à l'Aude et au Roussillon : $101 \mathrm{~km} / \mathrm{h}$ au Cap-Béar (Pyrénées-Orientales), $104 \mathrm{~km} / \mathrm{h}$ à Beauvais-Tillé (Oise) sous orage et $115 \mathrm{~km} / \mathrm{h}$ à Ouessant-Stiff (Finistère) le 21 ; $101 \mathrm{~km} / \mathrm{h}$ à Labruguière (Tarn) le 22 .

Du 24 au 26 ainsi que le 29, de fortes rafales de sud-ouest à ouest intéressent les côtes de la Bretagne et de la Manche : $115 \mathrm{~km} / \mathrm{h}$ à Belle-Ille-le-Talut (Morbihan) le $25 ; 108 \mathrm{~km} / \mathrm{h}$ à la Pointe-deChémoulin (Loire-Atlantique) le 26.

Du 23 au 29, le mistral souffle quotidiennement ; il est rejoint par la tramontane du 23 au 25 et le 27 ainsi que par un flux de sud-ouest en Corse du 26 au 28 : $108 \mathrm{~km} / \mathrm{h}$ au Cap-Béar (PyrénéesOrientales) le $23 ; 108 \mathrm{~km} / \mathrm{h}$ au CapCorse (Haute-Corse) le $27 ; 108 \mathrm{~km} / \mathrm{h} \mathrm{au}$ Cap-Cépet (Var) le 28.

Le nombre de jours avec vent fort atteint un maximum de 24 au CapBéar (Pyrénées-Orientales).
Écart à la normale de la température moyenne (degrés Celsius)

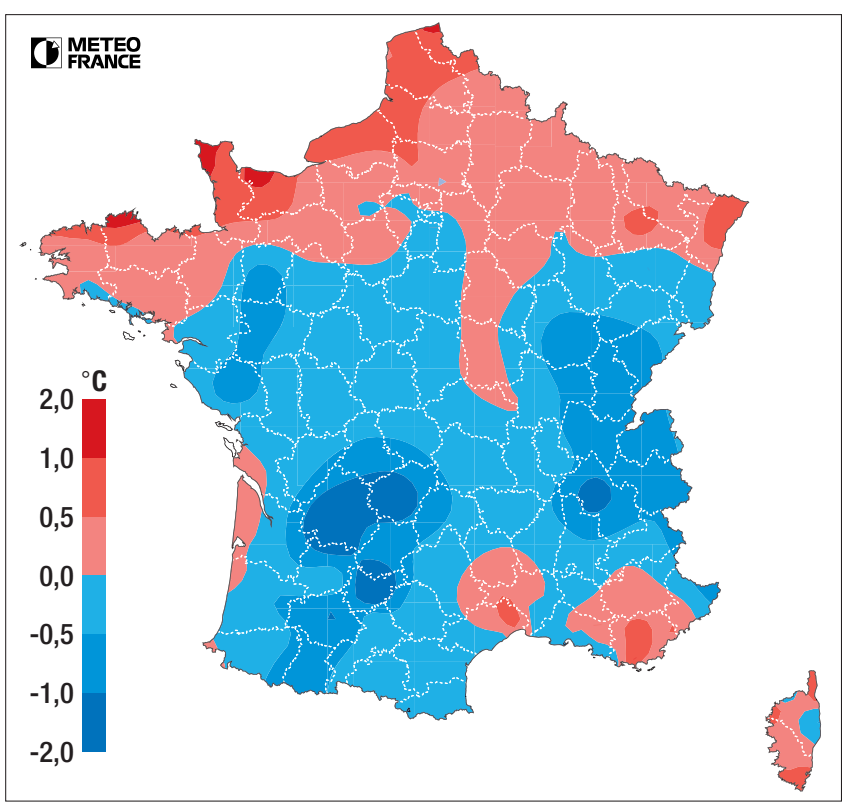

\section{Rapport à la normale des hauteurs de précipitations} (pour cent)

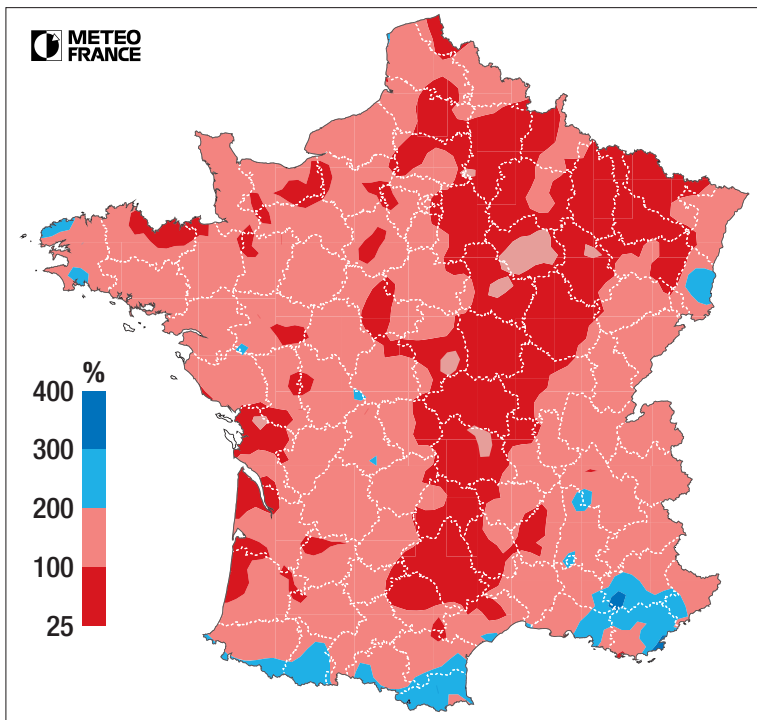

